\title{
On security of a Certificateless Aggregate Signature Scheme
}

\author{
Limin Shen, Yinxia Sun \\ Jiangsu Engineering Research Center of Information Security and Privacy Protection Technology \\ School of Computer Technology, Nanjing Normal University, Nanjing 210097, China \\ Email: shenlimin@njnu.edu.cn
}

\begin{abstract}
Aggregate signatures are useful in special areas where the signatures on many different messages generated by many different users need to be compressed. Very recently, Xiong et al. proposed a certificateless aggregate signature scheme provably secure in the random oracle model under the Computational Diffie-Hellman assumption. Unfortunately, by giving two kinds of concrete attacks, we indicate that the certificateless aggregate signature scheme of Xiong et al. does not meet the basic requirement of unforgeability.
\end{abstract}

Keywords: Aggregate signature, Certificateless aggregate signature, Unforgeability, Computational Diffie-Hellman problem

\section{Introduction}

In traditional public key cryptography, the authenticity of public keys is ensured by certificates signed by a certificate authority (CA). But the issues associated with certificate management are quite complex and costly. In 1984, Shamir [1] first invented a new paradigm called Identity Based public key cryptography (ID-PKC) which eliminates the need for certificate by deriving public keys for users directly from their identity information, such as e-mail address, telephone number or other identifiers. However, in ID-PKC a trusted third party called Private Key Generator (PKG) must be employed to generate the user's private key. Clearly, a malicious PKG can decrypt any ciphertext and forge the signature of any user. So ID-PKC suffers from an inherent problem namely key escrow.

To overcome the key escrow problem of ID-PKC, Al-Riyami and Paterson [2] proposed a new notion called certificateless public key cryptography (CL-PKC) in 2003. CL-PKC effectively solves the inherent key escrow problem in ID-PKC while keeps its certificate-free property. Similar to ID-PKC, CL-PKC also uses a third party called Key Generation Center (KGC) to help a user to generate his private key. However, KGC can only provide the partial private key for the user. The full private key is finally generated by the user who makes use of the secret value chosen by himself and the partial private key obtained from the KGC. The public key of the user is computed from his secret value and the KGC's public parameters, and it is published by the user himself. One of the most important primitives in public key cryptography is digital signature. Designing a high secure and efficient signature scheme is always desirable since we sometimes have to work in environments with low computability, low-bandwidth communication and low-storage. And an aggregate signature scheme is a signature scheme in which multiple signatures can be compressed into one single signature. The validity of an aggregate signature will convince a verifier that the $n$ users did indeed sign the $n$ original messages. The advantages 
of CL-PKC, such as escrow-free and certificate-free, may enable the wide use of certificateless signatures (CLS). In fact, there may be some cases of synchronously transmitting and verifying many different signatures signed by many signers. So, it is natural to consider to extend the notion of aggregate signatures to certificateless public key settings to get certificateless aggregate signatures (CLAS), which can aggregate many different certificateless signatures into one single signature, and effectively reduce the message size and verification cost. So, it is interesting to study secure and efficient constructions of aggregate signatures in CL-PKC.

The aggregate signature scheme was proposed by Boneh et al. [3] in which many different messages generated by many different signatures can be compressed into one single signature. Since then, many papers in this area have been published in traditional PKC [4-6]. The first Identity-based aggregate signature (IDAS) scheme was presented by Cheon et al. [7]. Later Cheng et al. [8], Xu et al. [9], Gentry and Ramzan [10], and Selvi et at. [11] introduced some efficient IDAS schemes, respectively. In recent years, much attention has been paid to the study of CL-PKC. So, the concept of certificateless aggregate signature (CLAS) has been introduced and several CLAS schemes have been proposed [12-15]. Very recently, Xiong et al. [16] introduced a new efficient certificateless aggregate signature scheme in which the signers do not need to be synchronized and its performance is comparable to the most efficient up-to-date schemes. They also claimed that their scheme was provably secure under the computational Diffie-Hellman assumption in the random oracle model. Unfortunately, their Security proof is not sound and their scheme is in fact insecure.

In this paper, we show that the CLAS scheme in [16] is flawed by demonstrating two kinds of attacks against it. In our first attack, we show that a Type I adversary who replaces one user's public key of an aggregating set $\mathbb{U}$ can forge a valid aggregate signature to a receiver. Similarly, in the second attack, we show that a Type II adversary who knows master key may impersonate any identity of an aggregating set $\mathbb{U}$ to generate a valid aggregate signature. Therefore, the scheme [16] is subject to the universal forgery of Type I and Type II adversaries. Thus, the original CLAS scheme of Xiong et al. [16] fails to achieve the security goal for an aggregate signature scheme.

The rest of this paper is organized as follows. Section 2 gives some preliminaries. Section 3 introduces the definition and the security notions for CLAS schemes. Section 4 reviews the CLAS scheme of Xiong et al. [16]. And Section 5 presents the attacks on Xiong et al.'s scheme. Finally, Section 6 concludes this paper.

\section{Preliminaries}

This section revisits some basic concepts and necessary complexity assumptions.

\section{$2.1 \quad$ Bilinear pairing}

Let $G_{1}$ and $G_{2}$ denote two multiplicative cyclic groups of prime order $q$, and $g$ be a generator of $G_{1}$. A map $\hat{e}: G_{1} \times G_{1} \longrightarrow G_{2}$ is called a bilinear pairing if it satisfies the following properties: - Bilinearity: $\hat{e}\left(g^{a}, h^{b}\right)=\hat{e}(g, h)^{a b}$ for all $g, h \in G_{1} a, b \in Z_{q}^{*}$.

- Non-degeneracy: $\hat{e}(g, g) \neq I_{G_{2}}$, where $I_{G_{2}}$ is the identity element of $G_{2}$.

- Computability: There exits an efficient algorithm to compute $\hat{e}(g, h)$ for all $g, h \in G_{2}$. 


\subsection{Complexity Assumptions}

Discrete Logarithm (DL) Problem: Given a generator $g$ of a cyclic group $G$ with order $q$, and $h \in G$ to find an integer $a \in Z_{q}^{*}$ such that $h=g^{a}$.

The DL assumption means that there is no polynomial time algorithm to solve the DL problem in $(g, h, G)$ with non-negligible advantage.

Computational Diffie-Hellman (CDH) problem: Given a generator $g$ of a cyclic group $G$ with order $p$, and given $\left(g^{a}, g^{b}\right)$ for unknown $a, b \in Z_{q}^{*}$, to compute $g^{a b}$.

The CDH assumption means that there is no polynomial time algorithm to solve the CDH problem in $\left(g, g^{a}, g^{b}, G\right)$ with non-negligible advantage.

\section{Certificateless Aggregate Signature(CLAS)}

\subsection{Formal Definition of Certificateless Aggregate Signature schemes}

A generic certificateless aggregate signature scheme is defined by six algorithms[14,16]: MasterKeyGen, Partial-Private-Key-Extract, UserKeyGen, Sign, Aggregate and Aggregate Verify. The description of each algorithm is as follows.

MasterKeyGen: This algorithm is performed by KGC that accepts a security parameter $k$ to generate a master key and a list of system parameters params.

PartialPrivateKeyGen: This algorithm is performed by KGC that accepts a user's identity $I D_{i}$, a parameter list params and a master key to produce the user's partial private key $p s k_{I D_{i}}$.

UserKeyGen: An algorithm run by a user that takes as input the user's identity $I D_{i}$, and selects a random $x_{I D_{i}} \in Z_{q}^{*}$, outputs the user's secret/public key pair $\left(u s k_{I D_{i}}, u p k_{I D_{i}}\right)$.

Sign: An algorithm run by each user $U_{i}$ in an aggregating set $\mathbb{U} . U_{i}$ 's inputs are the parameter list params, his identity $I D_{i}$, a signing key $\left(p s k_{I D_{i}}, u s k_{I D_{i}}\right)$ and a message $m_{i} \in\{0,1\}^{*}$. The output of $U_{i}$ is a signature $\sigma_{i}$.

Aggregate: An algorithm run by the aggregate signature generator that takes as inputs an aggregating set $\mathbb{U}$ of $n$ users $\left\{U_{1}, \cdots, U_{n}\right\}$, the identity $I D_{i}$ of each user $U_{i}$, the corresponding public key $u p k_{I D_{i}}$ of $U_{i}$, and a signature $\sigma_{i}$ on a message $m_{i}$ under identity $I D_{i}$ and public key $u p k_{I D_{i}}$ for each user $U_{i} \in \mathbb{U}$. The output of this algorithm is an aggregate signature $\sigma$ on messages $\left\{m_{1}, \cdots, m_{n}\right\}$.

Aggregate Verify: This algorithm takes as input an aggregating set $\mathbb{U}$ of $n$ users $\left\{U_{1}, \cdots, U_{n}\right\}$, the identity $I D_{i}$ and a corresponding public key $u p k_{I D_{i}}$ of each user $U_{i}$, an aggregate signature $\sigma$ on messages $\left\{m_{1}, \cdots, m_{n}\right\}$. It outputs true if the aggregate signature is valid, or false otherwise.

\subsection{Security requirements of Certificateless Aggregate Signature}

The basic security requirements for an aggregate signature scheme is unforgeability. Intuitively, we say that an aggregate signature scheme offers unforgeability if nobody can generate a valid aggregate signature on behalf of a generator without the possession of the full private key at least one of the users. Precise definitions of unforgeability is defined using security models. For the detail, please refer to $[14,16]$.

As defined in $[14,15,17]$, there are two types of adversary with different capabilities. 
Type I Adversary: This type of adversary $\mathcal{A}_{I}$ models a malicious adversary which does not have access to the master key, but $\mathcal{A}_{I}$ has the ability to replace the public key of any entity with a value of his choice, because there is no certificate involved in CLAS schemes.

Type II Adversary: This type of adversary $\mathcal{A}_{I I}$ has access to the master key but can not perform public key replacement.

\section{Revisiting the CLAS scheme of Xiong et al.}

In this section, we will review the CLAS scheme of Xiong et al. [16]. Let $G_{1}$ and $G_{2}$ denote two multiplicative cyclic groups of prime order $p$ and let $\hat{e}: G_{1} \times G_{1} \longrightarrow G_{2}$ be a bilinear pairing.

MasterKeyGen: Given a security parameter $k \in Z$, the algorithm works as follows:

1) Run the parameter generator on input $k$ to generate a prime $q$, two groups $G_{1}$ and $G_{2}$ of prime order $q$, two different generators $P$ and $Q$ in $G_{1}$ and an admissible paring $\hat{e}: G_{1} \times G_{1} \longrightarrow$ $G_{2}$.

2) Select a master key $s \in_{R} Z_{q}^{*}$ and set $P_{p u b}=s P$.

3) Choose cryptographic hash functions $H_{0}, H_{0}^{\prime}:\{0,1\}^{*} \rightarrow G_{1}$ and $H_{1}, H_{2}, H_{2}^{\prime}:\{0,1\}^{*} \rightarrow$ $Z_{q}^{*}$. The security analysis will review $H_{1}$ and $H_{2}$ as random oracles. The system parameters are $\left\{q, G_{1}, G_{2}, \hat{e}, P, Q, P_{p u b}, H_{0}, H_{0}^{\prime}, H_{1}, H_{2}, H_{2}^{\prime}\right\}$. The master key is $s$.

PartialPrivateKeyGen: Given a user's identity $I D_{i} \in\{0,1\}^{*}$, KGC first computes

$$
Q_{I D_{i}}=H_{0}\left(I D_{i}\right), Q_{I D_{i}}^{\prime}=H_{0}^{\prime}\left(I D_{i}\right)
$$

It then sets this user's partial key $p s k_{I D_{i}}=\left(s Q_{I D_{i}}, s Q_{I D_{i}}^{\prime}\right)$ and transmits it to user $I D_{i}$ secretly.

UserKeyGen: The user $I D_{i}$ selects a secret value $x_{I D_{i}} \in_{R} Z_{q}^{*}$ as his secret key $u s k_{I D_{i}}$, and computes his public key as $u p k_{I D_{i}}=x_{I D_{i}} P$.

Sign: Given its signing key $\left(u s k_{I D_{i}}, p s k_{I D_{i}}\right)$, and a message $m_{i} \in\{0,1\}^{*}$, the signer, whose identity is $I D_{i}$ and the corresponding public key is $u p k_{I D_{i}}$ performs the following steps.

1) Compute

$$
h_{i 1}=H_{1}\left(m_{i}, I D_{i}, u p k_{I D_{i}}\right), h_{i 2}=H_{2}\left(m_{i}, I D_{i}, u p k_{I D_{i}}\right), h_{i 2}^{\prime}=H_{2}^{\prime}\left(m_{i}, I D_{i}, u p k_{I D_{i}}\right)
$$

2) Compute

$$
\sigma_{i}=h_{i 1} \cdot x_{I D_{i}} \cdot Q+h_{i 2} \cdot s Q_{I D_{i}}+h_{i 2}^{\prime} \cdot s Q_{I D_{i}}^{\prime}
$$

3) Output $\sigma_{i}$ as the signature on $m_{i}$.

Aggregate: Anyone can act as an aggregate signature generator who can aggregate a collection of individual signatures. For an aggregating set $\mathbb{U}$ of $n$ users $\left\{U_{1}, \cdots, U_{n}\right\}$ with identities $\left\{I D_{1}, \cdots, I D_{n}\right\}$ and the corresponding public keys $\left\{u p k_{1}, \cdots, u p k_{n}\right\}$, and message-signature pairs $\left(m_{1}, \sigma_{1}\right), \cdots,\left(m_{n}, \sigma_{n}\right)$ from $\left\{U_{1}, \cdots, U_{n}\right\}$ respectively, the aggregate signature generator computes $\sigma=\sum_{i=1}^{n} \sigma_{i}$ and output $\sigma$ as an aggregate signature.

Aggregate Verify: To verify an aggregate signature $\sigma$ signed by $n$ users $\left\{U_{1}, \cdots, U_{n}\right\}$ with identities $\left\{I D_{1}, \cdots, I D_{n}\right\}$ and the corresponding public keys $\left\{u p k_{1}, \cdots, u p k_{n}\right\}$ on messages $\left\{m_{1}, \cdots, m_{n}\right\}$, the verifier performs the following steps.

1) For $i=1, \cdots, n$, compute

$$
Q_{I D_{i}}=H_{0}\left(I D_{i}\right), Q_{I D_{i}}^{\prime}=H_{0}^{\prime}\left(I D_{i}\right)
$$




$$
h_{i 1}=H_{1}\left(m_{i}, I D_{i}, u p k_{I D_{i}}\right), h_{i 2}=H_{2}\left(m_{i}, I D_{i}, u p k_{I D_{i}}\right), h_{i 2}^{\prime}=H_{2}^{\prime}\left(m_{i}, I D_{i}, u p k_{I D_{i}}\right)
$$

2) Verify the equation

$$
\hat{e}(\sigma, P)=\hat{e}\left(\sum_{i=1}^{n} h_{i 1} u p k_{I D_{i}}, Q\right) \hat{e}\left(\sum_{i=1}^{n}\left(h_{i 2} Q_{I D_{i}}+h_{i 2}^{\prime} Q_{I D_{i}}^{\prime}\right), P_{p u b}\right)
$$

If it holds, accept the signature; else reject it.

\section{$5 \quad$ Security Analysis of the CLAS Scheme by Xiong et al.}

In this section, we describe our attacks on Xiong et al.'s scheme [16] to show its security vulnerabilities. Let $\left\{U_{1}, \cdots, U_{n}\right\}$ be an aggregating set of $n$ users with identities $\left\{I D_{1}, \cdots, I D_{n}\right\}$ and the corresponding public keys $\left\{u p k_{1}, \cdots, u p k_{n}\right\}$ on messages $\left\{m_{1}, \cdots, m_{n}\right\}$.

\subsection{An Attack on Xiong et al.'s scheme Using Type I Adversary}

As defined in $[14,15]$, a certificateless aggregate signature scheme is existentially unforgeable iff it resists against type I and type II adversaries. Recall that a type I adversary $\mathcal{A}_{I}$ does not posses the knowledge of the master key, but the adversary can perform public key replacement, i.e. replacing the public key with his choice. We will show that the scheme in [16] does not resist against a type I adversary since the adversary can successfully forge a valid aggregate signature by replacing one user's public key. The concrete attack is described in four stages.

Stage 1: In this stage, $\mathcal{A}_{I}$ randomly chooses an identity, without losing generality, $\mathcal{A}_{I}$ chooses $I D_{n}$ and picks $x_{I D_{n}}^{\prime} \in \in_{R} Z_{q}^{*}$, computes $u p k_{I D_{n}}^{\prime}=x_{I D_{n}}^{\prime} P$, and replaces the $I D_{n}$ 's public key $u p k_{I D_{n}}$ with $u p k_{I D_{n}}^{\prime}$.

Stage 2: $\mathcal{A}_{I}$ queries the Super-Sign oracle for the signature of $m_{i}$ for $I D_{i}(i=1, \cdots, n-1)$. Let $\left\{\sigma_{1}, \cdots, \sigma_{n-1}\right\}$ be the output of the Super-Sign oracle.

Stage 3: $\mathcal{A}_{I}$ picks $m, m^{\prime} \in\{0,1\}^{*}\left(m, m^{\prime} \neq m_{i}, i=1, \cdots, n\right)$, queries the Super-Sign oracle for the signatures of $\left(I D_{n}, m\right)$ and $\left(I D_{n}, m^{\prime}\right) .\left\{\sigma_{n}, \sigma_{n}^{\prime}\right\}$ be the output of the Super-Sign oracle. Then $\mathcal{A}_{I}$ computes

$$
g_{n 1}=H_{1}\left(m, I D_{n}, u p k_{I D_{n}}^{\prime}\right), g_{n 2}=H_{2}\left(m, I D_{n}, u p k_{I D_{n}}^{\prime}\right), g_{n 2}^{\prime}=H_{2}^{\prime}\left(m, I D_{n}, u p k_{I D_{n}}^{\prime}\right)
$$

and

$$
k_{n 1}=H_{1}\left(m^{\prime}, I D_{n}, u p k_{I D_{n}}^{\prime}\right), k_{n 2}=H_{2}\left(m^{\prime}, I D_{n}, u p k_{I D_{n}}^{\prime}\right), k_{n 2}^{\prime}=H_{2}^{\prime}\left(m^{\prime}, I D_{n}, u p k_{I D_{n}}^{\prime}\right)
$$

So, $\mathcal{A}_{I}$ has the equations

$$
\left\{\begin{array}{l}
\sigma_{n}=g_{n 1} \cdot x_{I D_{n}}^{\prime} \cdot Q+g_{n 2} \cdot s Q_{I D_{n}}+g_{n 2}^{\prime} \cdot s Q_{I D_{n}}^{\prime} \\
\sigma_{n}^{\prime}=k_{n 1} \cdot x_{I D_{n}}^{\prime} \cdot Q+k_{n 2} \cdot s Q_{I D_{n}}+k_{n 2}^{\prime} \cdot s Q_{I D_{n}}^{\prime}
\end{array}\right.
$$

$\mathcal{A}_{I}$ can easily obtain the solutions $s Q_{I D_{n}}, s Q_{I D_{n}}^{\prime}$ from the equations. Then $\mathcal{A}_{I}$ computes

$$
h_{n 1}=H_{1}\left(m_{n}, I D_{n}, u p k_{I D_{n}}^{\prime}\right), h_{n 2}=H_{2}\left(m_{n}, I D_{n}, u p k_{I D_{n}}^{\prime}\right), h_{n 2}^{\prime}=H_{2}^{\prime}\left(m_{n}, I D_{n}, u p k_{I D_{n}}^{\prime}\right)
$$


and

$$
\sigma_{n}^{*}=h_{n 1} \cdot x_{I D_{n}}^{\prime} \cdot Q+h_{n 2} \cdot s Q_{I D_{n}}+h_{n 2}^{\prime} \cdot s Q_{I D_{n}}^{\prime}
$$

Stage 4: $\mathcal{A}_{I}$ generates an aggregate signature

$$
\sigma^{*}=\sum_{i=1}^{n-1} \sigma_{i}+\sigma_{n}^{*}
$$

Here, $\sigma_{n}^{*}$ is a forged signature of $\left(I D_{n}, m_{n}\right)$ and is not the output of the Super-Sign oracle.

Since we have

$$
\begin{aligned}
& \hat{e}\left(\sigma^{*}, P\right) \\
= & \hat{e}\left(\sum_{i=1}^{n-1} \sigma_{i}+\sigma_{n}^{*}, P\right) \\
= & \hat{e}\left(\sum_{i=1}^{n-1} h_{i 1} u p k_{I D_{i}}, Q\right) \hat{e}\left(h_{n 1} u p k_{I D_{n}}^{\prime}, Q\right) \\
& \hat{e}\left(\sum_{i=1}^{n-1}\left(h_{i 2} Q_{I D_{i}}+h_{i 2}^{\prime} Q_{I D_{i}}^{\prime}\right), P_{p u b}\right) \hat{e}\left(\left(h_{n 2} Q_{I D_{n}}+h_{n 2}^{\prime} Q_{I D_{n}}^{\prime}\right), P_{p u b}\right) \\
= & \hat{e}\left(\sum_{i=1}^{n} h_{i 1} u p k_{I D_{i}}, Q\right) \hat{e}\left(\sum_{i=1}^{n}\left(h_{i 2} Q_{I D_{i}}+h_{i 2}^{\prime} Q_{I D_{i}}^{\prime}\right), P_{p u b}\right)
\end{aligned}
$$

the verification equation always holds. This declares that the forged aggregate signature $\sigma^{*}$ is valid. Therefore, the scheme is subject to universal forgery with respect to a Type I adversary $\mathcal{A}_{I}$ who replaces one of the identities's public key.

\subsection{An Attack on Xiong et al.'s scheme Using Type II Adversary}

Recall that a type II adversary $\mathcal{A}_{I I}$ is given the master key, but can not replace any public keys. In most security models, $\mathcal{A}_{I I}$ is just like a "malicious - but-passive" KGC. We will show that the scheme in [16] does not resist against a type II adversary since $\mathcal{A}_{I I}$ can successfully forge a valid aggregate signature. The concrete attack is described in four stages.

Stage 1: In this stage, $\mathcal{A}_{I I}$ randomly chooses an identity, without losing generality, $\mathcal{A}_{I I}$ chooses $I D_{n}$ and picks $m_{n}^{\prime} \in\{0,1\}^{*}\left(m_{n}^{\prime} \neq m_{i}, i=1, \cdots, n\right)$. $\mathcal{A}_{I I}$ queries the Super-Sign oracle for the signature of $m_{n}^{\prime}$ for $I D_{n}$. Let $\sigma_{n}^{\prime}$ be the output of the Super-Sign oracle.

Stage $2: \mathcal{A}_{I I}$ computes

$$
k_{n 1}=H_{1}\left(m_{n}^{\prime}, I D_{n}, u p k_{I D_{n}}\right), k_{n 2}=H_{2}\left(m_{n}^{\prime}, I D_{n}, u p k_{I D_{n}}\right), k_{n 2}^{\prime}=H_{2}^{\prime}\left(m_{n}^{\prime}, I D_{n}, u p k_{I D_{n}}\right)
$$

So, $\mathcal{A}_{I}$ has the equation

$$
\sigma_{n}^{\prime}=k_{n 1} \cdot x_{I D_{n}} \cdot Q+k_{n 2} \cdot s Q_{I D_{n}}+k_{n 2}^{\prime} \cdot s Q_{I D_{n}}^{\prime}
$$

Since $\mathcal{A}_{I I}$ kmows the master key, $s$, it can easily obtain the solution $\left(x_{I D_{n}} \cdot Q\right)$ from the equation. Then $\mathcal{A}_{I I}$ computes

$$
h_{n 1}=H_{1}\left(m_{n}, I D_{n}, u p k_{I D_{n}}\right), h_{n 2}=H_{2}\left(m_{n}, I D_{n}, u p k_{I D_{n}}\right), h_{n 2}^{\prime}=H_{2}^{\prime}\left(m_{n}, I D_{n}, u p k_{I D_{n}}\right)
$$

and

$$
\sigma_{n}=h_{n 1} \cdot\left(x_{I D_{n}} \cdot Q\right)+h_{n 2} \cdot s Q_{I D_{n}}+h_{n 2}^{\prime} \cdot s Q_{I D_{n}}^{\prime}
$$

Here, $\sigma_{n}$ is a forged signature of $\left(I D_{n}, m_{n}\right)$ and is not the output of the Super-Sign oracle.

Stage 3: $\mathcal{A}_{I I}$ queries the Super-Sign oracle for the signature of $m_{i}$ for $I D_{i}(i=1, \cdots, n-1)$. Let $\left\{\sigma_{1}, \cdots, \sigma_{n-1}\right\}$ be the output of the Super-Sign oracle. 
Stage 4: $\mathcal{A}_{I I}$ generates an aggregate signature

$$
\sigma^{*}=\sum_{i=1}^{n} \sigma_{i}
$$

It is clear that $\sigma^{*}$ is a valid aggregate signature since the verification equation always holds:

$$
\hat{e}\left(\sigma^{*}, P\right)=\hat{e}\left(\sum_{i=1}^{n} h_{i 1} u p k_{I D_{i}}, Q\right) \hat{e}\left(\sum_{i=1}^{n}\left(h_{i 2} Q_{I D_{i}}+h_{i 2}^{\prime} Q_{I D_{i}}^{\prime}\right), P_{p u b}\right)
$$

\section{Conclusion}

In this paper, we demonstrated two kinds of concrete attacks against the recently proposed CLAS scheme by Xiong et al. according to their security model. In our attacks, a Type I adversary can forge a valid aggregate signature by replacing the public key of one of the identities, and a Type II adversary can forge a valid aggregate signature by using the master key. Thus, the CLAS scheme by Xiong et al. fails to meet the requirement of unforgeability for a secure aggregate signature scheme.

\section{References}

1. Adi Shamir. Identity-based cryptosystems and signature schemes. In CRYPTO, pp47-53, 1984.

2. S. S. Al-Riyami and K. G. Paterson. Certificateless Public Key Cryptography. Advances in CryptographyAsiacrypt 2003, LNCS 2894, Springer-Verlag, Berlin, pp452-473, 2003.

3. D. Boneh, C. Gentry, B. Lynn, H. Shacham, Aggregate and verifiably encrypted signatures from bilinear maps, in: E. Biham (Ed.), EUROCRYPT 2003, LNCS 2656, Springer-Verlag, Warsaw, Poland, pp416-432, 2003.

4. A. Lysyanskaya, S. Micali, L. Reyzin, H. Shacham. Sequential aggregate signatures from trapdoor permutations, in: C. Cachin, J. Camenisch (Eds.), Eurocrypt 2004, LNCS 3027, Springer-Verlag, Interlaken, Switzerland, pp74-90, 2004.

5. S. Lu, R. Ostrovsky, A. Sahai, H. Shacham, and B. Waters. Sequential Aggregate Signatures and Multisignatures Without Random Oracles, in Proc. Eurocrypt, Springer, pp465-485, 2006.

6. J. H. Ahn, M. Green and S. Hohenberger. Synchronized Aggregate Signatures: New Definitions, Constructions and Applications, in ACM Computer and Communications Security (CCS 2010), ACM, pp473-484, 2010.

7. J. Cheon, Y. Kim, H. Yoon, A new id-based signature with batch verification, Cryptology ePrint Archive, Report 2004/131.

8. X. Cheng, J. Liu, L. Guo, X. Wang, Identity-based multisignature and aggregate signature schemes from m-torsion groups, Journal of Electronics (China) 23(2006) 569573.

9. J. Xu, Z. Zhang, D. Feng, ID-based aggregate signatures from bilinear pairings, in: Y.G. Desmedt et al. (Eds.), CANS 2005, LNCS 3810, Springer-Verlag, Shenzhen, China, 2005, pp110-119.

10. C. Gentry, Z. Ramzan, Identity-based aggregate signatures, in:M. Yung et al. (Eds.), PKC 2006, LNCS 3958, Springer-Verlag, New York, USA, 2006, pp257-273.

11. D Selvi, S. Vivek, J. Shriram, Identity Based Aggregate Signcryption Schemes[C], B. Roy and N. Sendrier (Eds.): INDOCRYPT 2009, LNCS 5922, Springer-Verlag Berlin Heidelberg 2009, pp378-397, 2009.

12. R. Castro, R. Dahab, Efficient Certificateless Signatures Suitable for Aggregation. [Online]. Available: http://eprint.iacr.org/2007/454.pdf

13. Z. Gong, Y. Long, X. Hong, K. Chen, Two certificateless aggregate signatures from bilinear maps, in: SNPD 2007, IEEE Press, Qingdao, China, 2007, pp188-193.

14. L. Zhang, F. Zhang. A New Certificateless Aggregate Signature Scheme. Computer Communications, vol. 32, no. 6, pp1079-1085, 2009. 
15. L. Zhang, B. Qin, Q. Wu, F. Zhang. Efficient Many-to-One Authentication with Certificateless Aggregate Signatures. Computer Networks, vol. 54, no. 14, pp2482-2491, 2010.

16. Hu Xiong, Qianhong Wu, Zhong Chen. Strong Security Enabled Certificateless Aggregate Signatures Applicable to Mobile Computation. 2011 Third International Conference on Intelligent Networking and Collaborative Systems, 978-0-7695-4579-0/11 \$26.00@2011 IEEE DOI 10.1109/INCoS.2011.151.

17. K. Y. Choi, J. H. Park, D. H. Lee. A New Provably Secure Certificateless Short Signature Scheme. Computers and Mathematics with Applications, vol. 61, no. 7, pp1760-1768, 2011. 